\title{
Study of the degradation of the geometry of the railway between the pk 80 and 105 at the plateau of Settat (Morocco)
}

\section{Etude de la dégradation de la géométrie de la voie ferrée entre les PK80 et 105 au niveau du plateau de Settat (Maroc)}

\author{
Mohamed BEN OUAKKASS, Latifa OUADIF, Ahmed AKHSSAS et Lahcen BAHI \\ 3GIE Laboratory, Mohammadia Engineering School, Mohammed V University in Rabat-Morocco
}

\begin{abstract}
The plateau of Settat is formed by Paleozoic age formations surmounted by a subhorizontal cover made up mainly of Cretaceous sedimentary rocks formed of limestones, sandstones and clays with alternating marly limestones and marls. The study area is characterized by a dry to semi-dry climate, an endorheic hydrographic network that flows into the plain, and is affected by a hydraulic fracturing network of WNW-ESE and NE-SW directions. These factors cause repetitive and cyclical disturbances and disorders recorded on the railway section between Settat and Machraa Ben Abbou and are due to the shrinkage-swelling of the clay formations. The statistical and critical analysis of the defects identified by the device of measuring the geometric parameters of the railway, coupled with an exploitation of the Optical and Radar satellite images, made it possible to refine the geological, hydrological and structural study of the plain studied. These approaches have shown that this hazard has been favored by the existence of recent clayey and swelling formations under the influence by the climatic, hydrological and geological contrasts marked by intense hydraulic fracturing of the zone.
\end{abstract}

Keywords: Swelling clays, railway, remote sensing, radar and optical imagery, Settat, Morocco

Résumé. Le plateau de Settat est constitué par des formations d’âge paléozoïques surmonté par une couverture subhorizontale constituée essentiellement de roches sédimentaires du Crétacé formés de calcaires, grès et d'argiles avec une alternance de calcaires marneux et de marnes. La zone d'étude est caractérisée par un climat aride à semi-aride, par un réseau hydrographique endoréique qui se déverse dans la plaine, et elle est affectée par un réseau de fracturation de directions WNW-ESE et NE-SW, l'ensemble de ces facteurs provoque des dérangements et des désordres répétitifs et cycliques enregistrés sur le tronçon ferroviaire entre Settat et Machraa Ben Abbou de la voie ferrée qui sont dues au retrait-gonflement des formations argileuses. L'analyse statistique et critique des défauts relevés par le dispositif de mesure des paramètres géométriques de la voie, couplée d'une exploitation des images satellitaires Optique et Radar, ont permis d'affiner l'étude géologique, hydrologique et structurale de la plaine d'étude. Ces approches ont fait ressortir que cet aléa a été favorisé par l'existence des formations récentes argileuses et gonflantes sous l'influence des contrastes climatiques, hydrologiques et géologiques marquées par une fracturation intense de la zone.

Mots clé : Argiles gonflant, voie ferrée, télédétection, imagerie radar et optique, Settat, Maroc

\section{Introduction}

La qualité géométrique de la voie est l'évaluation des variations des paramètres spécifiés par rapport à la moyenne ou aux caractéristiques géométriques de construction, dans le sens vertical ou latéral, qui peuvent mettre en cause la sécurité de circulation des trains [1]

La dégradation de l'état géométrique de la voie dépend, principalement, de la nature et de l'importance du trafic, mais aussi d'autres facteurs locaux à savoir la 
nature des formations géologiques, les paramètres géotechniques et les conditions climatiques.

Bien que l'armement de la voie, la géométrie de pose et les sollicitations du trafic soient identiques sur une même section, le comportement des zones élémentaires est très différent.

Le tronçon objet de cette présente étude (Figure 1) fait partie administrativement à la province de Settat et situé entre la gare ferroviaire de Settat du point kilométrique (PK) 72 et la gare de Mechraa Ben Abbou du PK 117 de la ligne Casablanca à Marrakech sur une longueur d'environ de $25 \mathrm{~km}$.

La zone traversée actuellement par 18 trains voyageurs dont 16 trains reliant Marrakech à Casablanca et 2 trains Marrakech à Tanger, le trafic marchandise a permet de transporter 234467 tonnes en 2015. Avec les projets, en cours de réalisation, de doublement complet de la voie entre Casablanca et Marrakech et les zones logistiques de Sidi Bou Othman et Sidi Ghanem, l'offre ferroviaire sera augmentée aussi bien voyageurs que marchandises. Suite à des désordres géométriques répétitifs et cycliques enregistrés dans la zone d'étude, il était nécessaire d'en analyser les causes.

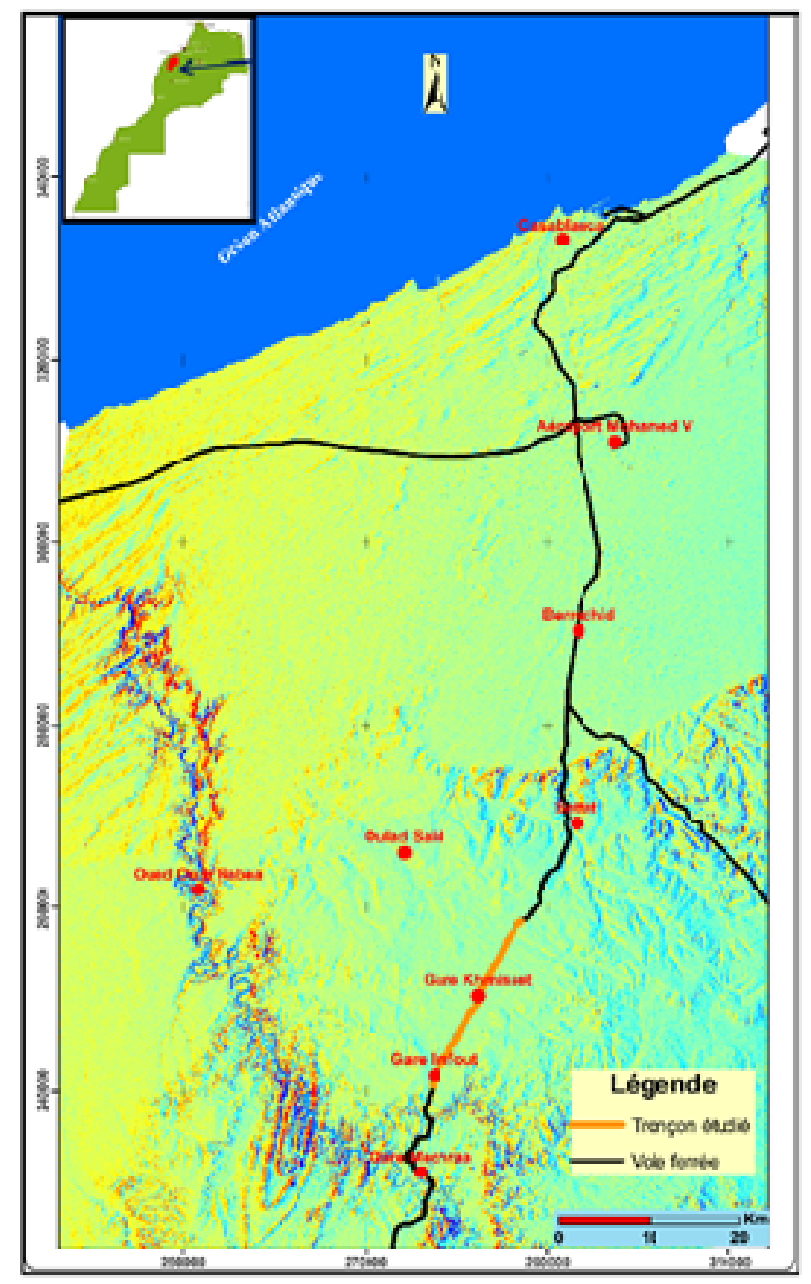

Fig. 1. Situation géographique de la zone d'étude

\section{Géométrie de la voie ferrée}

La géométrie de la voie se compose du tracé auquel sont superposés les défauts. Le référentiel de la qualité géométrique de la voie a pour objectif de définir les seuils des paramètres géométriques pour permettre une interprétation juste des amplitudes des défauts et des mesures à prendre et pour mieux adapter la nature des corrections à la situation et au degré de gravité des anomalies.

Le tracé de la voie est défini par quatre paramètres : la courbure horizontale, la courbure verticale, le dévers et l'écartement nominal. Quant aux défauts, ils en existent de quatre types suivants : le dressage, l'écartement par rapport à l'écartement nominal, le profil en long et le gauche.

\subsection{Défauts géométriques de la voie ferrée}

La qualité géométrique de la voie est décrite en utilisant un système orthogonal de coordonnées centré sur la voie. Les indicateurs de qualité de la géométriques sont comme suit [2] :

- Dressage : est le déplacement latéral de la voie dans le plan de roulement, l'écart par rapport à la position horizontale moyenne (flèche) est mesuré sur la base de $10 \mathrm{ml}$;

- Ecartement : c'est la distance entre les faces actives des deux files de rail, il est considéré comme étant la plus petite distance entre les rails ;

- Profil en long: correspond à la variation des altitudes consécutives de la table de roulement de chaque file de rail exprimée comme un écart par rapport à la position verticale moyenne (ligne de référence);

- Gauche : c'est la différence algébrique entre deux valeurs de dévers mesurées à une distance donnée, exprimée habituellement comme un gradient entre les deux points de mesure. Le gauche est exprimé en $\mathrm{mm} / \mathrm{m}$.

\subsection{Seuils paramètres géométrique de la voie}

Les seuils des paramètres géométriques de la voie sont définis par classe de voie et en fonction de la vitesse limite. Pour chaque paramètre quatre niveaux principaux sont définis [3] :

- $\quad$ Limite d'Alerte (LA) : correspondant à la valeur qui demande l'analyse de la géométrie de la voie et la prise en compte lors des opérations de maintenance programmées ;

- Limite d'intervention (LI) : correspondant à la valeur qui, lorsqu'elle est dépassée, demande une action de maintenance corrective telle que la limite d'action immédiate ne soit pas atteinte avant le prochain contrôle.

- Limite d'action immédiate (LAI) : Les défauts atteignant ou dépassant le seuil «LAI » nécessitent une limitation de vitesse des trains jusqu'à correction du défaut.

- Tolérance de réception (TR) : correspondant à une valeur qui ne doit pas être dépassée après des travaux de pose de voie nouvelle, de renouvellement de voie ou d'entretien de la voie.

Lors de la campagne de mesure, le système informatique de l'engin permet de suivre et de visualiser le déroulement de la mesure et de l'enregistrement de la 
qualité géométrique de la voie en temps réel sous forme des graphes (Figure 2), qui représentent toutes les dimensions de la géométrie de la voie.

\subsection{Mesure de paramètres géométriques de la voie}

Les paramètres géométriques de la voie sont mesurés par l'autorail qui permet de calculer des indicateurs représentatifs de la qualité de la voie [4]. C'est est un véhicule automoteur équipé d'un système informatique pour l'enregistrement des mesures et l'analyse des résultats. Le dispositif de mesure se compose d'une partie mécanique et d'une partie électrique.

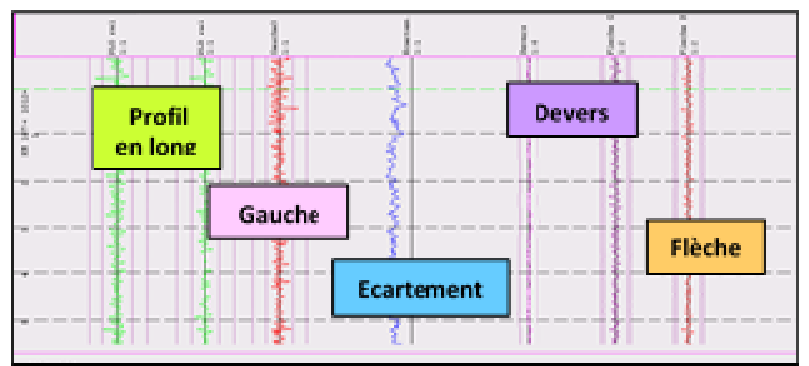

Fig. 2. Graphes de l'enregistrement des indicateurs de la qualité géométrique de la voie ferrée

La périodicité de mesure d'enregistrement est fixée comme suit [3] :

- Mesure et enregistrement en janvier ou février, après la période de froid, pour l'évaluation du bilan de l'année écoulée et élaboration des programmes d'entretien de la voie pour l'année en cours.

- Enregistrement en septembre, après la période de forte chaleur, pour l'appréciation de la qualité des interventions et ajustement des programmes d'entretien de la géométrie.

- Un troisième enregistrement effectué en mai pour l'appréciation de la qualité des interventions et ajustement des programmes d'entretien de la géométrie de la voie.

\section{Etude de la dégradation de la géométrie de la voie dans la zone entre Settat et Mechraa Ben Abbou}

A trafic donné, il en résulte que la vitesse de dégradation de la qualité géométrique d'une voie peut considérablement varier en fonction de facteurs locaux. L'objectif de l'entretien de la géométrie de la voie est de satisfaire aux conditions de sécurité et de confort des circulations et d'éviter l'aggravation des défauts dont la correction deviendrait par la suite plus difficile et onéreuse. La détection des déformations apparaît comme étant un paramètre essentiel pour l'aménagement et la gestion des risques, notamment en contexte ferroviaire où les enjeux sont importants.

\subsection{Analyse statistique géométriques de la voie}

des

défauts

Le rapport ponctuel édité en temps réel par l'engin de mesure de la qualité des paramètres géométriques permet de localiser pour chaque paramètre, les défauts dont l'amplitude atteigne ou dépasse le seuil limite d'action.

La zone d'étude a connu un renouvellement complet de la voie en 2010, la figure 3 représente les résultats de traitement des enregistrements trimestrielles des indicateurs de la géométrie de la voie, notamment le gauche, le flèche et le profil en long, entre 2010 et le deuxième trimestre de l'année 2017.

En tenant compte des opérations de correction des défauts effectuées entre les deux campagnes de mesure consécutives, on constate une variation du nombre moyen des défauts (Gauche, Flèche et profil en long) enregistrés d'un trimestre à l'autre. Nous remarquons aussi que le nombre moyen de défauts mesurés au troisième trimestre, correspondant à la période des fortes chaleurs, est plus élevé par rapport aux autres périodes.

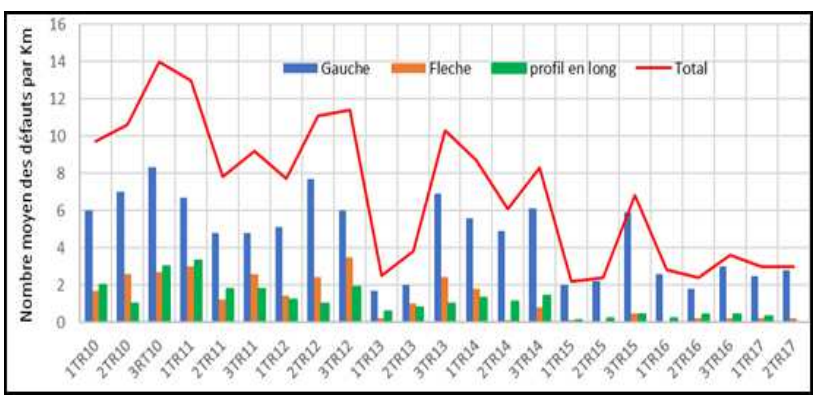

Fig. 3. Evolution de la qualité géométrique de la voie entre les PK 80 et 105 période : 2010-2017

Dans l'objectif d'étudier les variations de défauts géométrique et leur distribution dans l'espace le long de la voie, on a réparti ces anomalies selon les points kilométriques $(\mathrm{PK})$ où elles ont été mesurées. Les figures 4 , 5 et 6 représentent la situation géographique des défauts enregistrés par leurs PK et par trimestre entre les années 2012 et 2016. D'après ces représentations on peut distinguer trois zones selon le nombre des défauts enregistrés :

- Zone stable entre les points kilométriques PK 88 et PK 92 : correspond à la zone où on a enregistré moins de défauts, le nombre cumulé ne dépasse pas cinq défauts au premier trimestre et vingt au troisième trimestre ;

- Zones moyennement stables entre PK 80 et PK 87 et entre PK 96 et PK 99: dont le nombre cumulé de défaut mesuré est de l'ordre de 20 au premier trimestre et 40 au troisième trimestre ;

- Zone perturbante: dans cette zone, on trouve des nombres élevés de défauts qui dépassent 100 défauts au PK 96 et 140 défauts au PK 100.

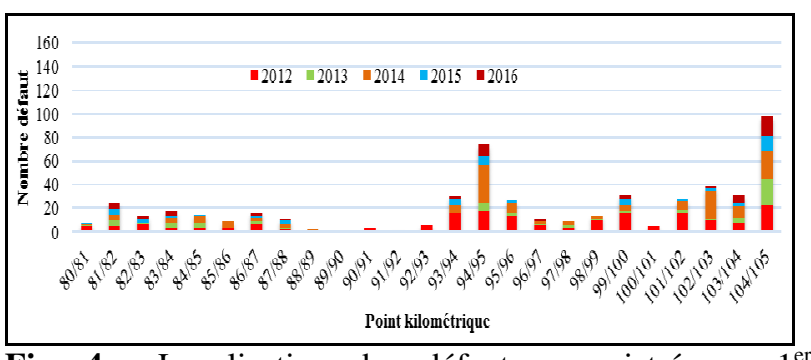

Fig. 4. Localisation des défauts enregistrés au $1^{\text {er }}$ trimestre entre PK 80 et PK 105 
D'après les résultats obtenus, les bilans de qualité et l'analyse de la distribution des anomalies dans la zone d'étude, on peut conclure que la dégradation de l'état de la qualité géométrique de la voie ferrée ne dépend pas seulement de conditions internes de la voie comme l'importance du trafic, l'état du ballast et la nature de l'armement de la voie. A cet effet, et pour déterminer l'origine de ces anomalies répétitives et les conditions de leur distribution spatiale, on a recouru aux paramètres extérieurs à la voie à savoir les conditions géologiques, structurales et climatiques.

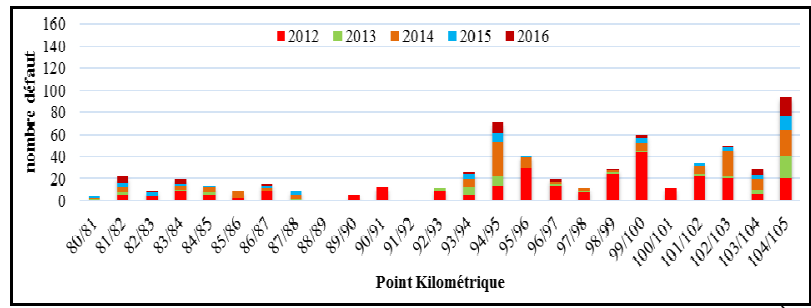

Fig. 5. Localisation des défauts enregistrés au $2^{\text {ème }}$ trimestre entre PK 80 et PK 105

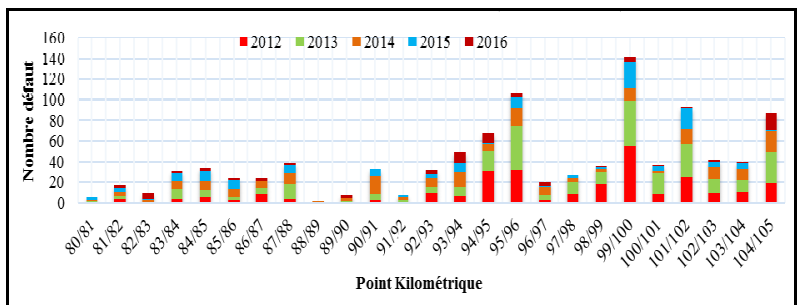

Fig. 6. Localisation des défauts enregistrés au $3^{\text {eme }}$ trimestre entre PK 80 et PK 105

\subsection{Climatologie et hydrologie de la région d'étude}

Le climat qui règne dans la région de Settat est de type aride à semi-aride [5] à hiver froid [6], la température moyenne annuelle de la région est de $18^{\circ} \mathrm{C}$. Le mois de janvier est le plus froid alors que le mois d'août est le plus chaud (Figure 7). On retrouve un gradient croissant des températures du Nord au Sud qui marque un degré de continentalité croissant.

La pluviométrie moyenne annuelle (Figure 7) à l'échelle de la province de Settat est de $372 \mathrm{~mm}$. La période pluvieuse s'étale d'octobre à avril, les mois de décembre, janvier et février sont les plus arrosés. La période estivale est pratiquement sèche avec des précipitations faibles à nulles, les variations interannuelles sont très marquées. Le niveau d'évapotranspiration annuelle est de $1350 \mathrm{~mm}$, il reste très élevé relativement à des précipitations de la région.

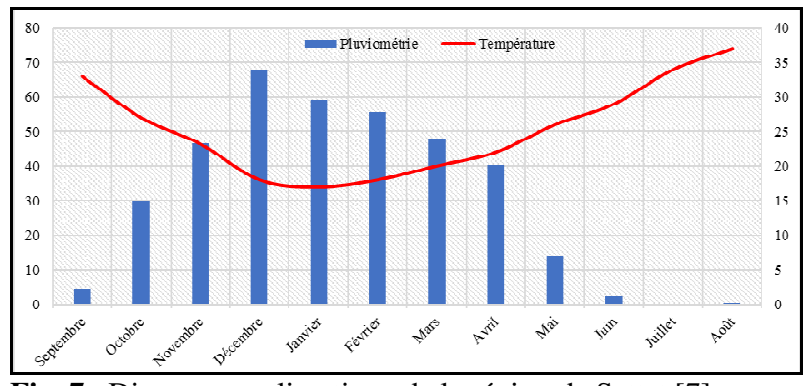

Fig. 7. Diagramme climatique de la région de Settat [7].
Le plateau de Settat est caractérisé par un réseau hydrographique endoréique [8] sous forme d'une série de petits cours d'eau de faible débit et à écoulement irrégulier. L'imagerie satellite optique et radar permet de mettre en évidence ces petits cours d'eau (Figure 8) de caractère saisonnier et de faible calibre qui se perdent en alimentant la nappe plio-quatenaire, ils ne possèdent aucun exutoire superficiel pour les eaux de pluie [9].

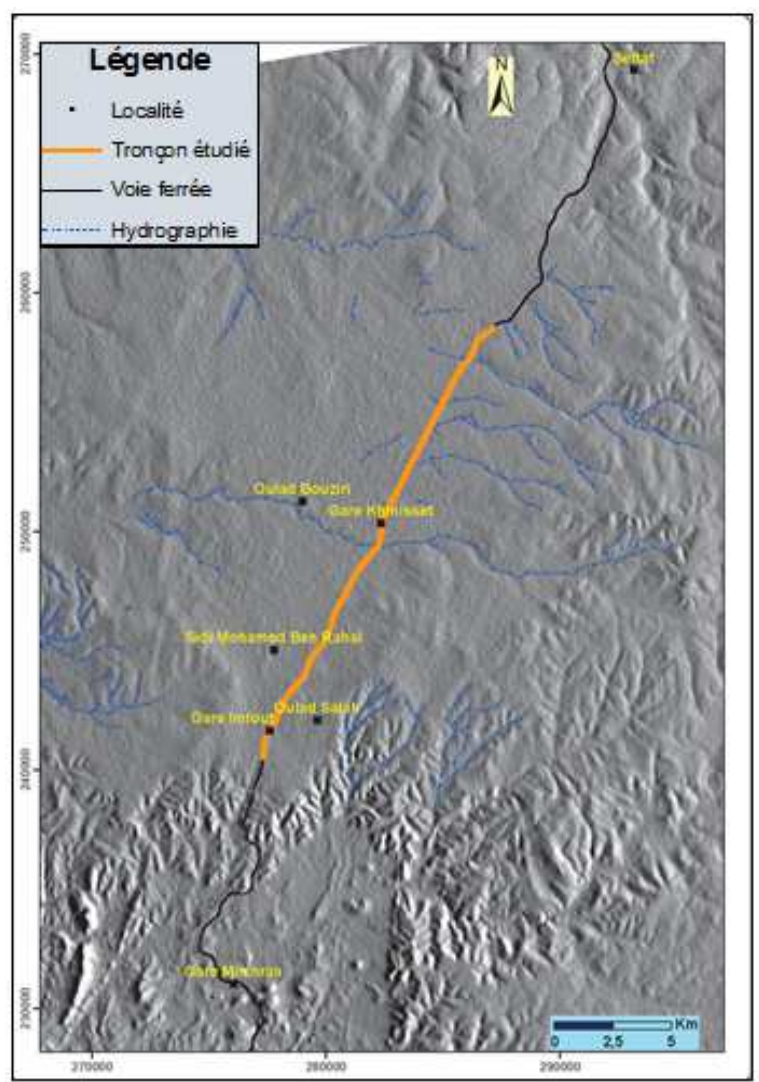

Fig. 8. Réseau hydrographique de la région d'étude

\subsection{Contexte géologique}

La région d'étude fait partie du plateau de Settat qui constitue la limite Nord-Ouest du plateau des phosphates de la Mesta centrale [10]. Celle-ci est constitué par des terrains du socle hercynien, plissés et granitisés arasés qui affleurent au $\mathrm{N}$ et au SW du plateau [11], ils sont recouverts par une couverture subtabulaire [12-13] peu déformés par les phases alpines [14], les formations superficielles quaternaires de la région résultent de l'action combinée des conditions climatiques et des mouvements tectoniques régis par le retrait du Moghrebiein [8] qui a donné naissance à une sédimentation continentale, au moment du soulèvement du plateau.

La série lithologique dans le plateau de Settat (Figure 9) débute par les formations d'âge paléozoïques, essentiellement représentées par des schistes, grès, calcaires récifaux, calcaires métamorphiques, conglomérats, grès quartzitiques et quartzites, plissées et faillées [5]. Le Primaire s'ennoie sous le haut plateau des phosphates [15], il affleure essentiellement au NordOuest du plateau de Settat, sur les bordures Sud-Est de la plaine de Berrechid, au Nord-Ouest du plateau de Ben Ahmed et au Sud aux environs Mechraa Ben Abbou. La 
couverture subhorizontale constituée essentiellement de roches sédimentaires du Crétacé (Cénomano-Turonien) formés de calcaires, grès et d'argiles avec une alternance de calcaires marneux et de marnes. Les couches les plus récentes sont datées de l'Eocène, l'ensemble de ces formations est couvert par une couche argilo-limoneuse du Quaternaire.

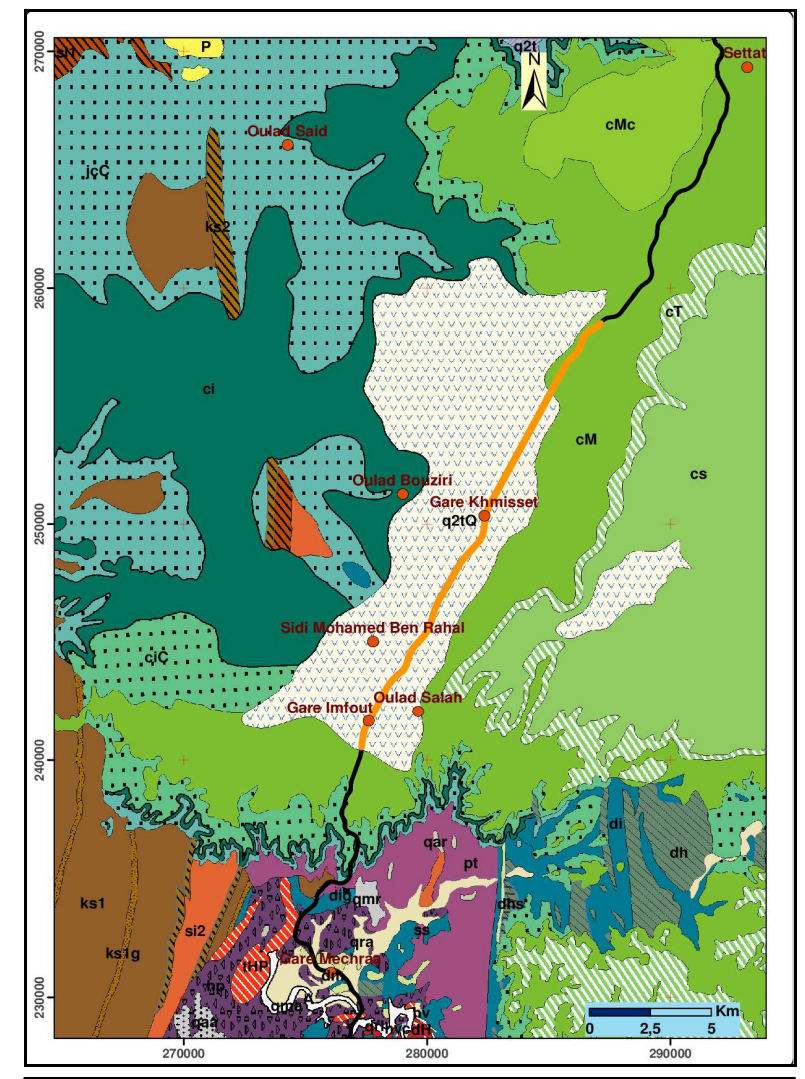

\begin{tabular}{|c|c|c|c|c|}
\hline \multicolumn{5}{|c|}{ Légende } \\
\hline P & Localité & 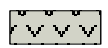 & $q 1 I Q$ & ds \\
\hline & Tronçon étudié & & $\mathrm{Pd}$ & di-c \\
\hline & Voie ferrée & & $\mathrm{P}$ & dig \\
\hline & $A$ & 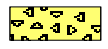 & $\mathrm{Pc}$ & di-s \\
\hline & q2a & 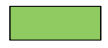 & cs & di \\
\hline & q2t & DN & cT & ss \\
\hline$\therefore$ & $\mathrm{q} 2 \mathrm{Fe}$ & L & cMc & si2 \\
\hline 18 & q21 & 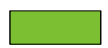 & $\mathrm{cM}$ & si1 \\
\hline$w$ & $\mathrm{q} 2 \mathrm{tQ}$ & $\because \cdot \cdot$ & $\mathrm{ciC}$ & ks2 \\
\hline 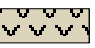 & $Q$ & & $\mathrm{ci}$ & ks1 \\
\hline & qra & 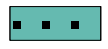 & $\mathrm{jcC}$ & ks1g \\
\hline 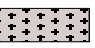 & qma & & $\mathrm{dh}$ & $x d$ \\
\hline & qmr & $\sqrt{1}$ & dhs & $x s i$ \\
\hline 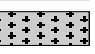 & qaa & & pt & $x k$ \\
\hline & qar & 104 & $\mathrm{hp}$ & BPT \\
\hline & e1 & $E$ & hvc & $\mathrm{dH}$ \\
\hline n & q1a & & hv & $\mathrm{tHP}$ \\
\hline m & q1c & & $\mathrm{dm}$ & \\
\hline
\end{tabular}

Fig. 9. Extrait des cartes géologiques au 1/200.000 de la meseta entre Settat et Mazagon (Dokkala et chaouia occidental) [16], du plateau du phosphate et la zone synclinale de Tadla [17] et de la meseta entre Mechra BenAbbou et Safi (Abda, Dokkala et Massif des Rehamna) [18] modifiées.
A : alluvions modernes ; $\mathrm{q} 2 \mathrm{a}:$ Terrasses limoneuses ; $\mathrm{q} 2 \mathrm{t}$ : Tirs ; $\mathrm{q} 2 \mathrm{Fe}$ : Limons à concrétions ferrugineuses; q21: limons rouges ; q2t ; Q : Croûte ; qra : Terrasses limoneuses ; qma/ q1a: Alluvions anciennes ; qmr: Eluvions; qar: Quaternaire ancien ; e1: Eocène inférieur série phosphatée ; q1c: Cônes d'éboulis et de déjections ; q11Q: Limons inferieurs encroûtés ; Pd: Dunes consolidées attribuées au Villafranchien ; $\mathrm{P}$ : Pliocène marin: grès coquilliers, mollasses, lumachelles ; Pc: Conglomérats fluvio-marins pliocènes ; cs : Sénonien : série marneuse ; cT: Turonien: calcaires ; cMc : Cénomanien calcaire ; cM : Cénomanien : série marno-calcaire ; ciC: Néocomien: argiles rouge ; ci : Néocomien marin calcaire ; jcC: Couches rouges de base (sommet de Jurassique) ; dh : Strunien : grès ; dhs : Strunien Schistes ; pt : Permo-Trias : argiles rouges, grès, conglomérats ; hp : Stéphano-Autunien : conglomérats ; hvc: Viséen supérieur : Calcaires ; dm : Dévonien moyen : Calcaires ; ds : Dévonien supérieur: schistes; di-c: Dévonien inferieur: Calcaires ; dig: Dévonien inferieur: Grès ; di-s : Dévonien inferieur : schistes ; di : Dévonien inferieur ; ss : Gothlandien : schistes à Graptolites ; si2 : Llandeilo : schistes, grès, quartzites ; si1: Arenig: Schistes ; ks2: Quartzites d'Imfout ; ks1 : Acadien: Schistes ; xd: Phyllandes d'origine dévonienne ; xsi : Phyllandes d'origine ordovienne ; xk: Phyllandes d'origine cambrienne ; BPT : Basaltes doléritiques du Trias ; dH : Dolérites hercyniennes ; tHP : Trachytes et andésites.

\subsection{Fracturation de la zone}

Le plateau de Settat a subi les effets de la compression alpine, dont les conséquences sont l'existence quasi permanente d'une légère flexion dans le secteur [5]. Les failles les plus importantes de la région sont de direction NNE-SSW à NE-SW, on trouve aussi une famille de failles d'orientation NW-SE caractérisées par une grande continuité sans décalage apparent, ces failles jouent un très grand rôle dans l'orientation de l'écoulement des oueds du plateau de Settat.

L'imagerie radar et optique fournit des informations fort utiles telle que l'identification des roches et des structures géologiques de surface [19-20], elle permet aussi de mesurer les déplacements de la surface de la terre potentiellement influencée par les changements dans l'humidité du sol [21]. La figure 10 représente le réseau de fracturation du secteur d'étude à l'aide de l'imagerie radar et optique. L'analyse de la rosace des directions de la fracturation montre trois principales directions : ENEWSW, NE-SW et NNE-SSW qui coïncident avec les familles des failles rencontrées dans l'ensemble de plateau de Settat.

\subsection{Analyse des sondages géotechnique}

Les neuf sondages carottés de $15 \mathrm{~m}$ de profondeur réalisés le long de la voie ferrée permettent de cerner la géologie locale en dégageant l'aspect structural de la zone d'étude ainsi que la nature des formations rencontrées (Figure 11).

En se basant sur les données de ces sondages, le sol en place est formé du marno-calcaire surmonté d'argile et limon tufacés et l'ensemble est coiffé de terre végétale ou remblai hétérogène. Dans le sondage $\mathrm{SC} 36$, on a rencontré le substratum sous forme de quartzite à partir de 13,80m de profondeur par rapport au terrain naturel.

Les sols susceptibles de se gonfler sont pour l'essentiel des argiles [22] suite à la succession de 
périodes humides et sèches en raison des variations saisonnières [23-24], les phénomènes de retraitgonflement des sols argileux et des formations géologiques argileuses induisent des tassements différentiels [25] qui se manifestent par l'instabilité des ouvrages de génie civil construits [26], l'apparition de fissures et autres désordres dans ces constructions [27], affaiblissement différentiel au-dessous des fondations peu profondes [28]. Les photos la figure 12.A illustrent les fissures au niveau des constructions situées à 100 de la voie ferrée dans le secteur d'étude et la figure 12.B représente les fissures et glissement de la chaussée de la route nationale $\mathrm{n}^{\circ} 9$ reliant Settat à Marrakech à $1 \mathrm{Km}$ environ de la voie ferrée.
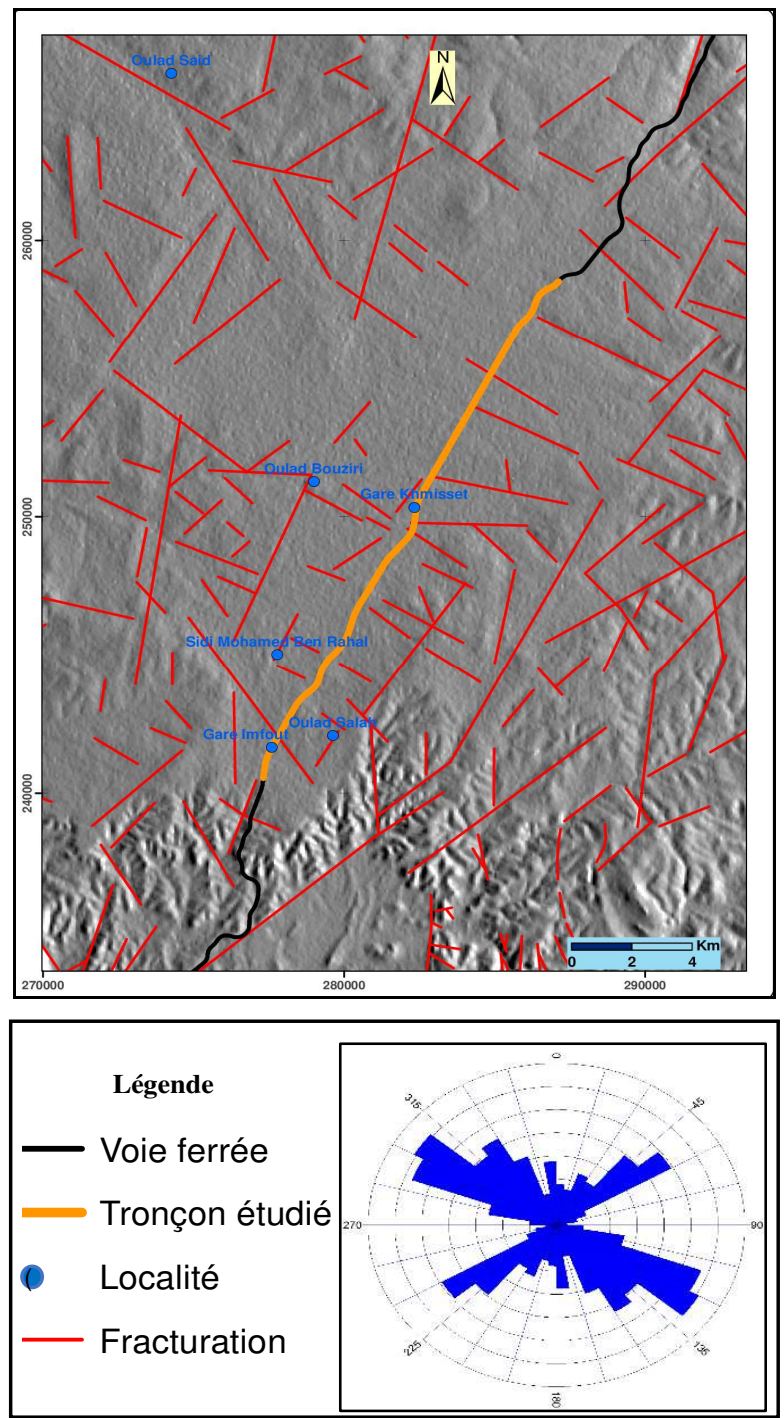

Fig. 10. Réseau et rosace de fracturation

Les problèmes dus aux mouvements des terrains suite à des cycles de retrait-gonflement, reconnues comme un risque naturel coûteux, sont présents partout dans le monde [29], l'abondance de ce sol expansif à l'échelle mondiale a généré trop d'efforts pour mieux comprendre leur comportement [30-31] et d'éviter toutes catastrophes qui peuvent survenir [32]. Ils sont associés à des situations déficientes en eau et dépend d'un ensemble de propriétés mécaniques et physicochimiques des matériaux [33, 34, 35]. C'est l'une des causes géotechniques les plus courantes [36], et qui a pour conséquence une modification des propriétés physiques du sol entraînant des mouvements de terrain de subsidence et de retrait-gonflement [37].
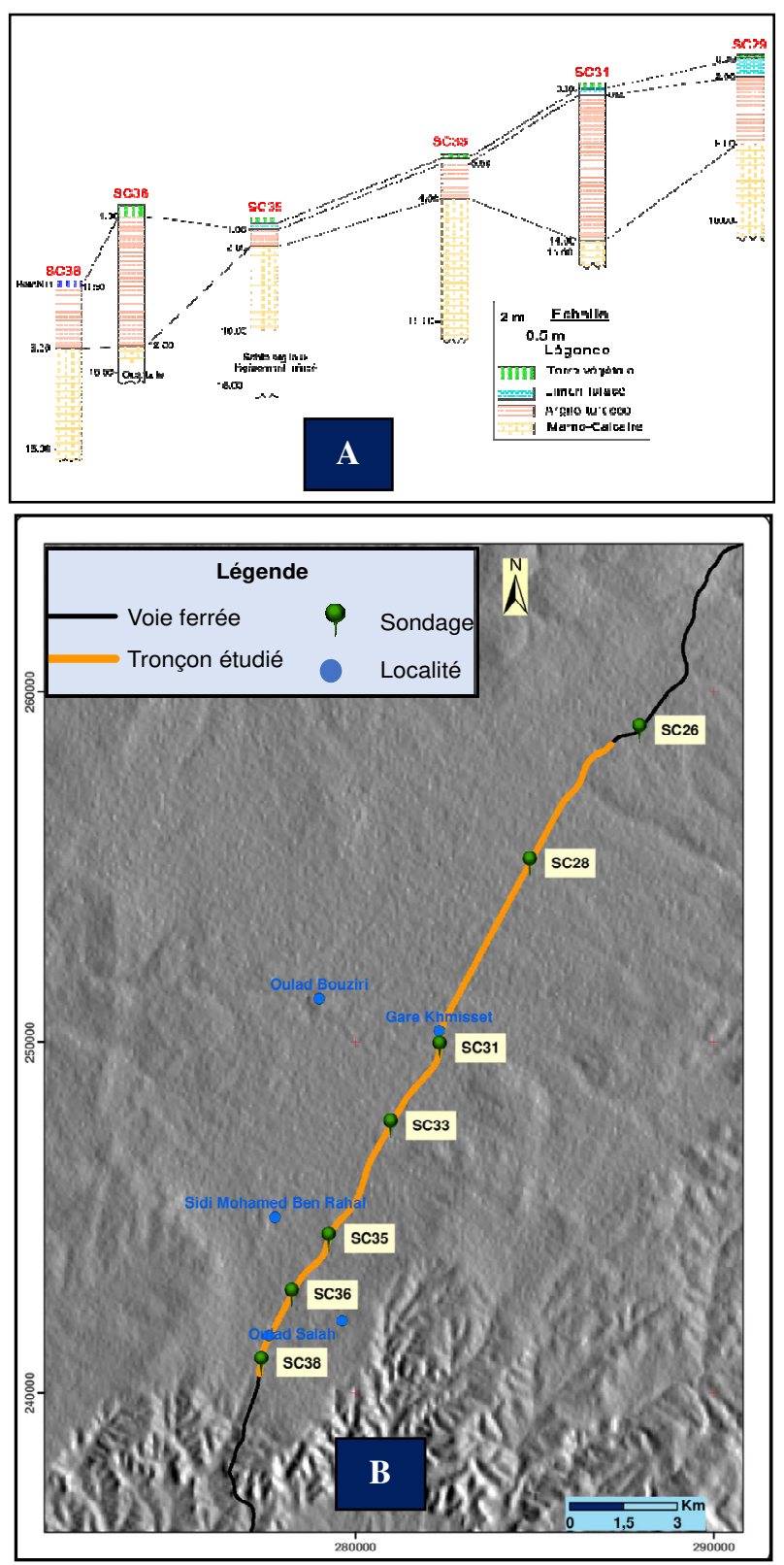

Fig. 11. A : Corrélation et variation latérale des formations à partir des sondages. B : Situation géographique des sondages carottés réalisés. 


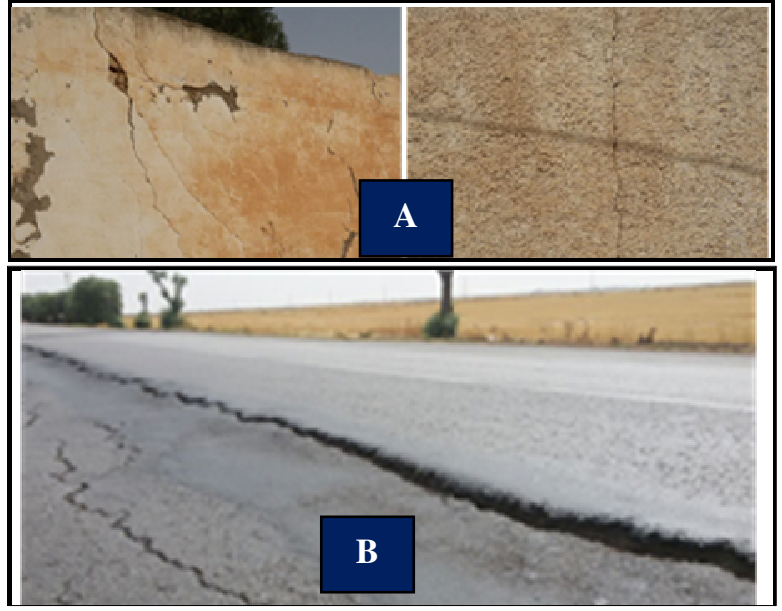

Fig. 12. A : Photos illustrant les fissures au niveau des constructions dans la zone d'étude. B : fissures et glissement de la chaussée sur la route nationale $\mathrm{n}^{\circ} 9$

Les formations argileuses et marno-calcaire de la région d'étude sont des matériaux très plastiques [8] et gonflants, qui peuvent causer des problèmes tels que les risques de tassement différentiel vertical et l'ouverture de fissures suite aux variations des teneurs en eau, ce qui favorise la dégradation de la qualité géométrique de la voie. L'aléa retrait-gonflement devra donc être pris en compte dans l'entretien de la voie et dans la planification de projets futurs dans la région.

\section{Conclusion}

La présente étude a permis d'établir une corrélation entre la dégradation de la qualité géométrique de la voie ferrée et les caractéristiques géotechnique et géologique des sols sous la plateforme ferroviaire. En effet, les formations géologiques du secteur sont constituées d'argiles et marno-calcaire, ce sont des matériaux très plastiques qui changent du volume selon les variations de la teneur en eau : retrait en période de sécheresse, puis gonflement au retour des pluies. Ce phénomène de retraitgonflement, qui est un risque naturel coûteux, provoque des tassements différentiels verticaux et l'ouverture de fissures qui se manifestent par des désordres affectant principalement les constructions et les infrastructures.

Par ailleurs, le climat aride à semi-aride de zone d'étude, le réseau hydrographie endoréique et les champs de fractures ont favorisé les processus de retraitgonflement des sols. On a constaté une augmentation de l'amplitude des indicateurs de la qualité géométrique de la voie dans la période d'été et dans zone à forte fracturation.

L'étude géologique, structurale et hydrologique complétée par les données de l'imagerie optique et radar ont permis de mettre en évidence de l'aléa gonflementrétrécissement qu'est à l'origine de la dégradation de la qualité géométrique de la voie ferrée, par les tassements différentiels verticaux et les pertes de ballast dans les fissures sous la plateforme. Ce phénomène présente un risque naturel qu'il faut prendre en considération dans les opérations de la maintenance et dans les projets futurs dans la région.

\section{Références bibliographiques}

1. L. Ricci, Modélisations discrètes et continues de la voie ferrée ballastées. Thèse de Doctorat, Institut Navier-Laboratoire Analyse des Matériaux et Identification (2006)

2. N. Guerin. Approche expérimentale et numérique du comportement du ballast des voies ferrées. Thèse de Doctorat en Structures et Matériaux, Ecole Nationale des Ponts et Chaussées (1996)

3. Office National des Chemins de Fer (ONCF). Instruction relative à la qualité géométrique de la voie. $\mathrm{N}^{\circ}$ d'identification : IN. DMI. R3 009/MVO (2010)

4. N. Rhayma, Contribution à l'évolution des méthodologies de caractérisation et d'amélioration des voies ferrées. Thèse de Doctorat en Génie Civil, Université Blaise Pascal - Clermont Ferrand II (2010).

5. E. El Gasmi, B. El Mansouri et M. Tammael. Les écoulements superficiels dans le plateau de SettatBan Ahmed et la plaine de Berrechid : Hydrographie Endoréique. ISSN 2351-8014 Vol. 9 No. 1, 40-53 (2014)

6. E. Hassoune, A. Bouzidi, Y. Koulali et D. Hadarbach, Effets des rejets liquides domestiques et industriels sur la qualité des eaux souterraines au nord de la ville de Settat (Maroc), Bulletin de l'Institut Scientifique, Rabat, section Sciences de la Vie, $\mathrm{n}^{\circ}$ 28, 61-71 (2006)

7. M. El Oumri, A. Osrhiri, R. Moussadek, Z. Moatamid, W. Goebel, A. Tikdirine et A. Ambri. Vocation agricole des terres de la zone de Settat Rapport et cartes-. Institut National de la Recherche Agronomique (2007)

8. K. El Bouqdaoui. Etude géologique et géotechnique de la Ville de Settat : cartographie géotechnique et substance utile de la Province de Settat, Thèse de Doctorat Géologie, Université Mohammed V, Rabat (1995)

9. G. Choubert et A. Faure-Muret. Plaines et bassins du Maroc atlantique. Ressources en Eau du Maroc Tome 2, Notes et Mémoires du Service Géologique du Maroc $\mathrm{N}^{\circ}$ 231, 1975 (1962)

10. J. P. Ruhard, Chaouia et Plaine de Berrechid. Ressources en Eau du Maroc Tome 2. Plaines et bassins du Maroc atlantique. Notes et Mémoires du Service Géologique du Maroc, 231, 197 p (1975)

11. C. Archambault, M. Combe. J.P. Ruchard, Le plateau des phosphates : ressources en eau. Notes et Mémoires du Service. Géologique du Maroc, 231, 239-258 (1975)

12. A. Piqué, Contribution à la géologie structurale des Rehamna (Meseta marocaine méridionale). Le matériel paléozoïque et son évolution hercynienne dans l'ouest du massif, Thèse de Doctorat en Géologie, l'Université Louis Pasteur de Strasbourg (1972)

13. E. El Gasmi, A. Mridekh, B. El Mansouri, M. Tammal et M. El Bouhaddioui. Apport des données géophysiques et géologiques à la mise en évidence 
de nouveaux éléments structuraux associés à la flexure de Settat (Maroc central), Bulletin de l'Institut Scientifique, Rabat, Sciences de la Terre, $\mathrm{n}^{\circ}$ 36, 109-121 (2014)

14. L. Mahdioui, H. Charchari, H. Allali, J. Naja et A. Bakkali. Characterization and development of agricultural land the watershed the Oued Bou Moussa, high Chaouia, Settat, Morocco, IRACST Engineering Science and Technology: An International Journal (ESTIJ), Vol.5, No.2 (2015)

15. A. Michard. Eléments de Géologie Marocaine. Notes et Mémoires du Service Géologique, 252, $426 \mathrm{p}$ (1976)

16. M. Gigout. Carte géologique de la Meseta entre Settat et Mazagon (Dokkala et chaouia occidental) au 1/200000, Notes et Mémoires du Service géologique du Maroc (1950)

17. G. Choubert. Carte géologique du plateau des phosphates et de la zone synclinale de Tadla, feuilles Ben Ahmed- El Brouj, au 1/200 000, Notes et Mémoires du Service géologique du Maroc $\mathrm{n}^{\circ} 137$ (1959)

18. M. Gigout. Carte géologique de la meseta entre Mechra BenAbbou et Safi (Abda, Dokkala et Massif des Rehamna) au 1/200 000, Notes et Mémoires du Service géologique du Maroc (1950)

19. T. Phan Trong, T. T. Mai, V. L. Ngo, Q. V. Hoang, V. H. Nguyen, V. T. Dinh, D. Closson et A. Ozer. Apport de la télédétection à l'étude des failles actives et des risques sismiques au nord du Viêt-Nam, Revue Télédétection, Vol. 7, n 1-2-3-4, p. 465-481 (2007)

20. N. Baghdadi, G. Grandjean, D. Lahondère, P. Paillou et Y. Lasn, Apport de l'imagerie satellitaire radar pour l'exploration géologique en zones arides, $C . R$. Geoscience 337, 719-728 (2005)

21. J. P. Magnan, H. Ejjaaouani, V. Shakhirev, S. Bensallam, Etude du gonflement et du retrait d'une argile. Bulletin des Laboratoires des Ponts et Chaussées, pp 155-170 (2013)

22. S. Zwieback, S. Hensley et I. Hajnsek, Assessment of soil moisture effects on L-band radar interferometry, Remote Sensing of Environment 164, 77-89 (2015)

23. M. Mrad, Modélisation du comportement hydromécanique des sols gonflants non saturés, Thèse de Doctorat en Génie Civil - Hydrosystèmes Géotechnique, École Nationale Supérieure de Géologie de Nancy (2005)

24. S. Bensallam, L. Bahi, M. Alaoui, H. Ejjaaouani, V. Shakhirev. The effect of surcharge pressure on the cyclic vertical deformations for expansive clayey soils in Morocco. Geosciences Journal Vol. 18, No. 1, p. 81 - 87, March 2014

25. H. Nowamooz, Retrait/gonflement des sols argileux compactes et naturels, Thèse de Doctorat en Génie Civil - Hydrosystèmes - Géotechnique, Institut National Polytechnique de Lorraine (2007)

26. A. Muzahim, J. C. Tourayb, F. Bergaya, Une argile modèle pour l'étude du gonflement des sols argileux : la laponite-Na de synthèse. C.R. Académie des sciences Paris, Sciences de la terre et des planètes, 329-242 (1999)

27. L. Makki, M. Duc, N. Droniuc, P. Reiffsteck, A. Maloula, J. P. Magnan, Essai de retrait pour une meilleure classification de la sensibilité des sols à la sécheresse, SEC2008, Magnan, Cojean, Cui et Mestat (ed.), Editions du LCPC, Paris (2008)

28. S. Bensallam, L. Bahi, H. Ejjaaouani, V. Shakhirev, Shrinkage Curve: Experimental Study and Modelling. IJE TRANSACTIONS A: Basics Vol. 25, No. 3, 203-210 (2012)

29. H. Nowamooz, Effective stress concept on multiscale swelling soils, Applied Clay Science 101, 205214 (2014)

30. E. Jahangir, Phénomènes d'interaction sol-structure vis-à-vis de l'aléa retrait-gonflement pour l'évaluation de la vulnérabilité des ouvrages. Thèse de Doctorat en Génie Civil - Hydrosystèmes - Géotechnique, Institut National Polytechnique de Lorraine (2011)

31. 27. S. Bensallam, L. Bahi, H. Ejjaaouani, V. Shakhirev, A New Shrinkage Curve Model, Applied to Moroccan Clayey Soil. International Journal of Geosciences, 3, 507-514 (2012)

32. T. El Bahlouli et L. Bahi, Study On The Swelling Behavior Of The Green Clay Of Gzenaya (Morocco) Compared With Test Conditions. International Journal of Engineering Research and Applications (IJERA), Vol. 2, Issue 6, pp.1413-1423 (2012)

33. S. Fityus, O. Buzzi, The place of expansive clays in the framework of unsaturated soil mechanics, Applied Clay Science 43, 150-155 (2009)

34. S. Bensallamn, L. Bahi, H. Ejjaaouani, V. Shakhirev, K. Rkha Chaham, Clay soil settlement: In-situ experimentation and analytical approach, Soils and Foundations, 54(2):109-115 (2014)

35. H. Ejjaaouani, Interactions des fondations et des sols gonflants : pathologie, calculs et études expérimentales, Thèse Doctorat, Spécialité : Géotechnique, Ecole Nationale des Ponts et Chaussées (2008)

36. S. G. Fityus, D. W. Smith, M. A. Allman, Expansive Soil Test Site Near Newcastle, Journal of Geotechnical and Geoenvironmental Engineering, Vol. 130, No. 7 (2004)

37. H. Kaveh, Apport de l'interférométrie radar (DinSAR et PSI) pour l'étude des effets de la sècheresse géotechniques : applications à l'Est de la région Îlede-France. Thèse de Doctorat en Sciences de l'Information Géographique, Université de MarneLa-Vallée - Paris Est (2010) 